\title{
Preoperative decompression and diagnosis of pancreatic head adenocarcinoma in a patient with Roux-en-Y gastric bypass by means of EUS-guided gastric pouch hepaticogastrostomy
}
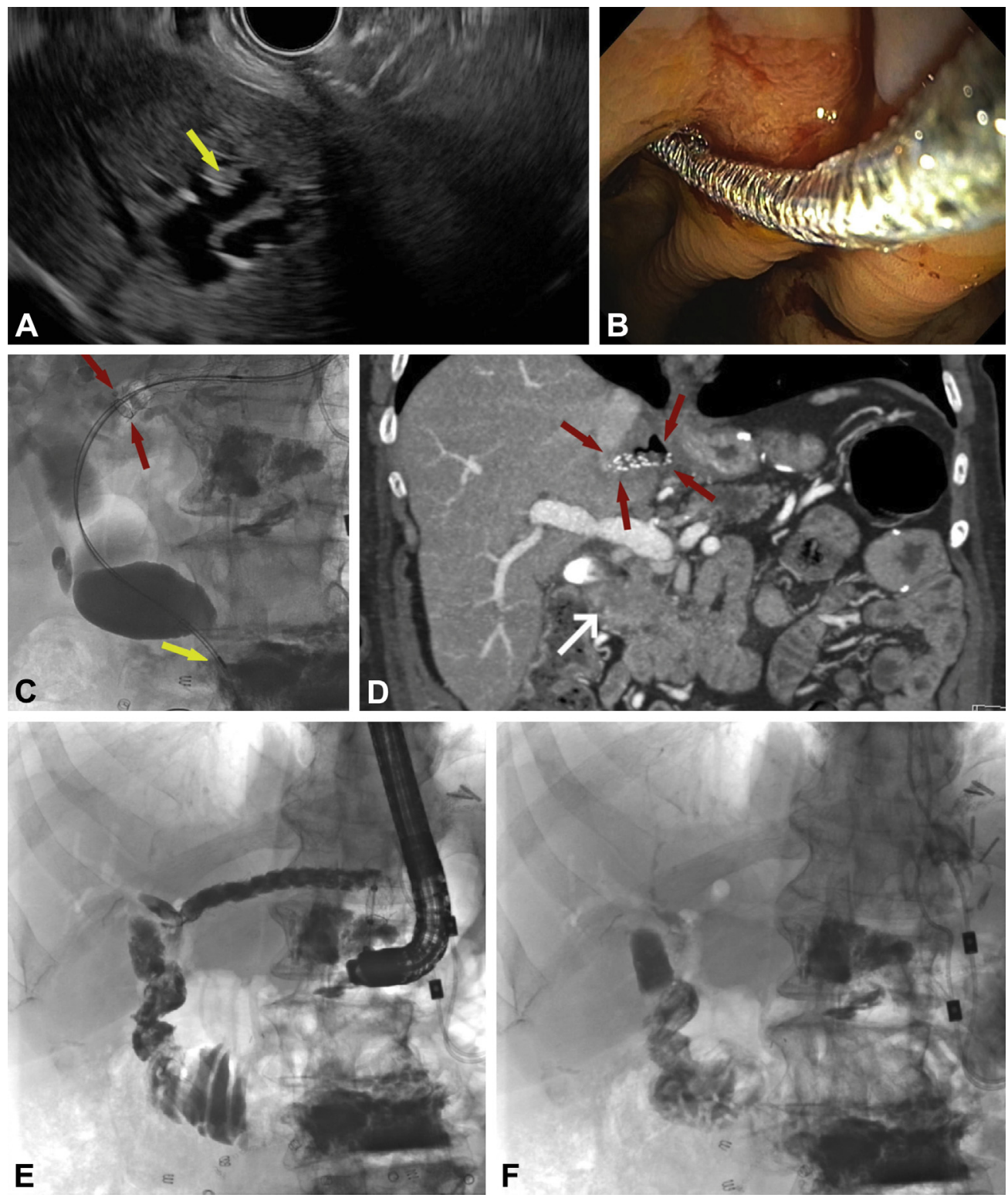

Figure 1. A, EUS image with the endoscope positioned in the gastric pouch shows dilated intrahepatic bile ducts (arrow). B, Endoscopic view of the proximal end of the hepaticogastrostomy stent from within the gastric pouch. C, Fluoroscopic image shows cytology brush positioned across the distal CBD stricture (yellow arrow) as it was passed antegrade via the HG stent (red arrows). D, Post-drainage, preoperative CT scan shows the HG stent in a good position (red arrows) and a resectable mass in the pancreatic head (white arrow). E, Fluoroscopic view of the HG stent 10 weeks after the Whipple procedure. F, Cholangiographic image obtained at the time of HG stent removal shows a patent choledochojejunostomy. $C B D$, common bile duct; $H G$, hepaticogastrostomy. 
A 74-year-old woman with prior Roux-en-Y gastric bypass (RYGB) presented to an outside facility with jaundice, anorexia, and weight loss. Her serum total bilirubin was $13 \mathrm{mg} / \mathrm{dL}$; abdominal CT showed a pancreatic head mass. She was referred for EUS/ERCP to decompress the biliary system and provide a diagnosis.

After discussing the alternatives, we performed EUSguided hepaticogastrostomy (HG) (Video 1, available online at www.VideoGIE.org). EUS from the gastric pouch showed dilated left intrahepatic bile ducts up to $10 \mathrm{~mm}$ in diameter. The left hepatic duct was punctured transgastrically from the pouch under EUS guidance with a 19G needle, followed by cholangiography showing a 20-mm distal common bile duct (CBD) stricture (Fig. 1A). A guidewire was passed antegrade into the duodenum. The HG was created by balloon dilation of the tract with a 4-mm balloon, followed by deployment of an $8-\mathrm{mm} \times 10-\mathrm{cm}$ long fully covered metal biliary stent from the left intrahepatic ducts to the gastric pouch under endoscopic and fluoroscopic guidance (Fig. 1B). The CBD stricture was sampled by brush cytology in an antegrade fashion (Fig. 1C). The procedure was completed with the patient as an outpatient, and there were no adverse events.

Postdrainage and preoperative triple-phase CT scanning showed a well-positioned HG stent and a resectable pancreatic head mass (Fig. 1D). Brush cytology at the time of HG was interpreted as suggestive of malignancy. Serum bilirubin decreased to $1.3 \mathrm{mg} / \mathrm{dL}$ by 4 weeks, followed by a Whipple procedure and removal of the excluded stomach. Surgical pathologic examination confirmed pancreatic adenocarcinoma. Ten weeks postoperatively, the HG stent was electively removed from the gastric pouch endoscopically; antegrade cholangiography through the
HG confirmed a patent choledochojejunostomy (Figs. 1E and $\mathrm{F}$ ). The patient remains clinically well.

This case shows that preoperative, single-session EUSguided HG allows antegrade cholangiography with brush cytology to assess distal bile duct obstruction in patients with RYGB. This may be the only endoscopic approach for tissue acquisition from the CBD or head of pancreas in RYGB patients that preserves the integrity of the Roux-en-Y bypass in patients who are reluctant to reverse the bypass with a gastro-gastric fistula, and it can be considered as an alternative to laparoscopicassisted ERCP, ERCP performed through gastrostomy tracks, and percutaneous drainage. Moreover, EUSguided HG does not preclude or interfere with subsequent Whipple operation.

\section{DISCLOSURE}

Dr Baron discloses financial relationships with W.L. Gore, Boston Scientific, and Cook Endoscopy. The other author disclosed no financial relationships relevant to this publication.

Mohamed M. Abdelfatah, MD, Division of Gastroenterology and Hepatology, East Carolina University, Greenville, North Carolina, USA, Todd H. Baron, MD, Division of Gastroenterology and Hepatology, University of North Carolina, Chapel Hill, North Carolina, USA

Copyright (c) 2017 American Society for Gastrointestinal Endoscopy. Published by Elsevier Inc. This is an open access article under the CC BY-NC-ND license (http://creativecommons.org/licenses/by-nc-nd/4.0/).

http://dx.doi.org/10.1016/j.vgie.2017.03.002 